EDITORIAL

\title{
Presentation of Volume 2, 1, 2020
}

\author{
José María Sarabia
}

Editor-in-Chief Spanish Journal of Statistics

Dear readers and dear statistical community:

It is my pleasure to present the second volume of the Spanish Journal of Statistics, corresponding to the year 2020. This volume includes four papers: two papers in the General Section and another two papers in the Official Statistics section.

The first paper is titled "Financial and Actuarial Properties of the Beta-Pareto as a Long-Tail Distribution" by Emilio Gómez-Déniz and Enrique Calderín-Ojeda. This paper presents several properties of the Beta-Pareto distribution, which might be extremely useful in Economics, and in Financial and Actuarial fields. These properties are mainly related to the analysis of the tail of the distribution that makes it a candidate model for fitting actuarial data with extreme observations.

The second article of the general section is titled: "The Gamma-Chen distribution: a new family of distributions with applications", by Lucas David R. Reis, Gauss M. Cordeiro and Maria do Carmo S. Lim. This paper presents the gamma-Chen distribution and derive some of its mathematical and statistical properties. The authors present empirical evidence that support that this new distribution is better than other relevant statistical distributions using several data sets.

The following two papers cover relevant aspects of Official Statistics. The first of them is: "Towards a modular end-to-end statistical production process with mobile network data", by David Salgado, Luis Sanguiao, Bogdan Oancea, Sandra Barragán, and Marian Necula. In the context of the European Statistical System (ESS), the authors introduce the so-called ESS Reference Methodological Framework for Mobile Network Data with the first modular and evolvable statistical process, which involves five different aspects: the geolocation of mobile devices; the deduplication of mobile devices; the statistical filtering to identify the target population; the aggregation into territorial units, and inference to the target population. The proposal methodology is illustrated with synthetic data generated from a network event data simulator developed for these purposes.

The second paper on Official Statistics is titled: "Commonly used methods for measuring output quality of multisource statistics", by Ton de Waal, Arnout van Delden and Sander Scholtus. The estimation of output quality based on sample surveys is well established. The paper presents results of the ESSnet project Quality of Multisource Statistics that studied methods to estimate output quality. The authors distinguish three main groups of methods: scoring methods, (re)sampling methods and methods based on parametric modeling. All of these methodologies are developed and 
discussed in detail within the paper.

I would like to conclude by thanking all the authors of this volume for choosing our journal as a means of disseminating their work.I am also extremely grateful to the editors and the reviewers of the papers for the work devoted to the journal, which is key to maintain a high scientific quality standard. 